\title{
Antisense expression of Gossypium barbadense UGD6 in Arabidopsis thaliana significantly alters cell wall composition
}

\author{
Jie Han ${ }^{1 \dagger}$, Yuxin $\operatorname{Pan}^{1,2 \dagger}$, Xingfen Wang ${ }^{1}$, Yan Zhang ${ }^{1} \&$ Zhiying $\mathrm{Ma}^{1 *}$ \\ ${ }^{1}$ North China Key Laboratory for Germplasm Resources of Education Ministry; Key Laboratory of Crop Germplasm Resources of Hebei \\ Agricultural University of Hebei, Baoding 071001, China; \\ ${ }^{2}$ North China University Science and Technology, Tangshan 063009, China
}

Received May 14, 2015; accepted July 20, 2015; published online January 22, 2016

\begin{abstract}
Uridine diphosphate-glucose dehydrogenase (UGD, EC1.1.1.22 oxidizes UDP-Glc (UDP-D-glucose) to UDP-GlcA (UDP-Dglucuronate), a critical precursor of cell wall polysaccharides. GbUGD6 from Gossypium barbadense is more highly expressed late in the elongation of cotton fibers (15 d post-anthesis (DPA)) and during the stage of secondary cell wall thickening (30 DPA). Subcellular localization analysis in onion epidermis revealed that fluorescently labeled GbUGD6 protein was distributed throughout the cell membrane, as well as the nucleus and vacuoles. Examination of UGD function in Arabidopsis revealed that the antisense GbUGD6 lines had shorter roots, deferred blossoming, compared to wild-type plants. Activities of associated enzymes were also affected by UGD reduction, and biochemical analysis of cell wall samples showed an increase in cellulose levels and a decrease in UGP-GlcA contents. The results of the present study as well as previous studies on UGD support the conclusion that UGD plays a major role in synthesizing polysaccharides synthesis in the cell wall.
\end{abstract}

UDP-glucose dehydrogenase, UDP-GlcA, Gossypium, fiber

Citation: Han, J., Pan, Y., Wang, X., Zhang, Y., and Ma, Z. (2016). Antisense expression of Gossypium barbadense UGD6 in Arabidopsis thaliana significantly alters cell wall composition. Sci China Life Sci 59, 213-218. doi: 10.1007/s11427-016-5004-y

\section{INTRODUCTION}

Uridine diphosphate-glucuronic acid (UDP-GlcA) is an essential precursor of eukaryotic hemicelluloses and pectin matrix polysaccharides (Diet et al., 2006; Karkonen et al., 2005; Malinova et al., 2014; Seifert, 2004) UDP-galacturonic acid (a pectin precursor) and UDP-xylose (precursor of hemicellulose) are formed through catalysis of UDPGlcA by UDP-d-glucuronic acid 4-epimerase (GAE) and UDP-glucuronic acid decarboxylase (UXS), respectively (Clough and Bent, 1998; Zablackis et al., 1995).

Synthesis of UDP-GlcA involves two pathways: nucleotide sugar oxidation (Kuhn et al., 2010) and myo-inositol oxidation (MIO) (Karkonen et al., 2005); however, it is un-

$\dagger$ Contributed equally to this work

*Corresponding author (email: mzhy@hebau.edu.cn) clear which is more important. Both utilize the substrate D-glucose-6-P. The nucleotide sugar oxidation (NSO) pathway utilizes three enzymes: phosphoglucomutase (PGM), UDP glucose pyrophosphorylase (UGP), and UDP-glucose dehydrogenase (UGD). The first two catalyze reversible reactions that form UDP-glucose (UDP-Glc), and as a key enzyme, UGD then catalyzes an irreversible reaction that converts UDP-Glc to UDP-GlcA (Diaz-De-Leon et al., 1993). The UGD enzyme has important functions in tobacco (Nicotiana tabacum) and Zea mays, and its differential expression has been described in Arabidopsis thaliana, poplar (Populus), soybean (Glycine max), and Phaseolus vulgaris (Bindschedler et al., 2005; Johansson et al., 2002; Karkonen et al., 2005; Klinghammer and Tenhaken, 2007; Robertson et al., 1996; Tenhaken and Thulke, 1996). In those cases, UGD expression is higher in 
young tissues and is also induced by biotic stress. In particular, down-regulation of UGD-A reduces pentose contents in the cell walls (Klinghammer and Tenhaken, 2007), which suggests that UGD has a critical role in the biosynthesis of UDP-GlcA and precursors of other cell wall polysaccharides.

In comparison, the MIO pathway involves five enzymatic steps that are catalyzed consecutively by myo-inositol phosphate synthase (MIPS; EC 5.5.1.4), myo-inositol monophosphatase (IMP; EC 3.1.3.25) and inositol oxygenase (MIOX; EC 1.13.99.1), glucuronokinase (EC 2.7.1.43), and UDP-glucuronic acid pyrophosphatase (EC 2.7.7.44). MIOX is the key enzyme in that pathway.

As valuable crops worldwide, various types of cotton (Gossypium spp.) have highly desirable fiber characteristics that are determined by matrix polysaccharides cross-linked by cellulose, hemicelluloses, and pectin. To improve their quality and yield, however, researchers must understand the mechanism by which those polysaccharides are formed. We previously used cDNA-AFLP technology to isolate a UGD fragment from $G$. barbadense (Pan, 2007). In the study described here, we cloned a full-length GbUGD6 gene that is preferentially expressed during the stages of late cotton-fiber elongation and secondary cell wall thickening. We then examined its functioning in Arabidopsis thaliana, and found that antisense expression of GbUGD6 changed the functioning of the above pathways, suggesting that the gene modulates the biosynthesis of cotton fibers.

\section{RESULTS}

\section{Features of GbUGD6 cDNA}

The reconstituted cDNA of GbUGD6 was 1,912 bp long,
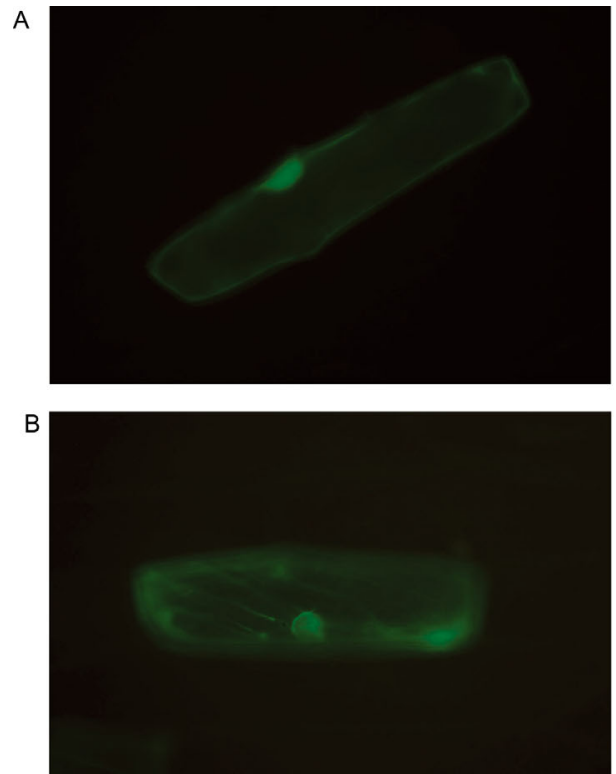

Figure 1 Subcellular localization of GbUGD6 fused with GFP in onion cells. A, Images of GFP in cells under confocal microscope (magnify 20×) (left, green fluorescence image; right, bright field image). B, Images of pCamE-UGD6::GFP expression vector in onion epidermal cells (magnify 20×) (left, green fluorescence image; right, bright field image).
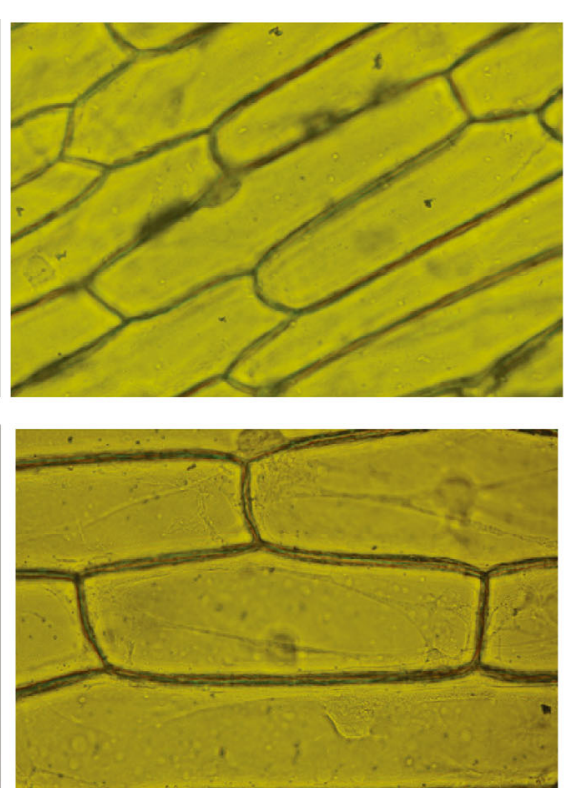

including a 1,443-bp open reading frame (ORF), a 168-bp 5'-untranslated region (UTR), and a 301-bp 3'-UTR. The ORF was determined to encode a peptide of 480 amino acid residues (Figure S1A in Supporting Information), and the deduced amino acid sequence was highly homologous to $A$. thaliana UGD catalytic subunits and differed from a previously cloned UGD (Figure S1 in Supporting Information) (Pang et al., 2010).

\section{Subcellular location of GFP-fused GbUGD6}

Although green fluorescent protein (GFP) signals from the empty vector control were detected in the cytomembrane and nucleus, pCamE- GbUGD6::GFP expression was observed in the cell membrane system, including the plasma membrane, nucleus, and vacuoles (Figure 1).

\section{mRNA expression profile for GbUGD6 in cotton fibers}

Results of our quantitative RT-PCR revealed similar GbUGD6 expression profiles for G. hirsutum ("CCRI 8") and G. barbadense ("Pima90-53"). In particular, transcripts accumulated sharply at 15 DPA, and then maintained a high level until 30 DPA (Figure S2 in Supporting Information). These patterns indicated that GbUGD6 expressed dramaticlly elongation of cotton fibers and during the stage of secondary cell wall thickening.

\section{Phenotypic alterations in transgenic Arabidopsis}

Phenotypic differences were obvious for growth habit and morphology between wild-type (WT) plants and antisense transgenic lines. After $7 \mathrm{~d}$ of development, roots of Arabidopsis plants from antisense transgenic lines were shorter than those of the WT (Figure 2). The WT plants began 


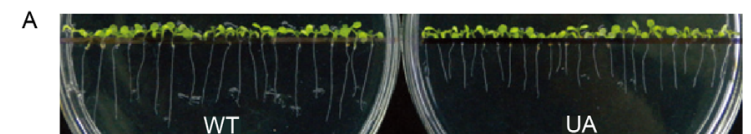

B

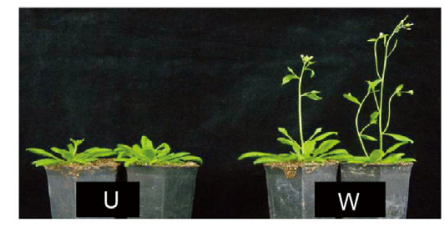

C

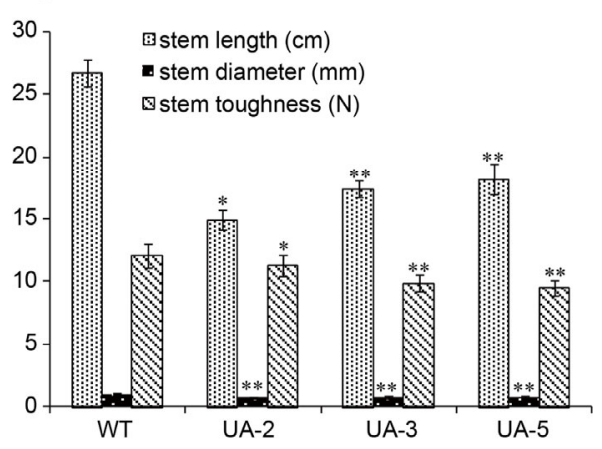

Figure 2 Phenotypes for wild-type (WT) and T3 transgenic (UA, GbUGD6-antisense) plants of Arabidopsis. A, Seedling roots from one week old seedlings. B, Shoot growth from 6-week-old seedlings. C, Phenotypic characters for wild-type (WT) and T3 transgenic (UA, GbUGD6-antisense) plants of Arabidopsis. **, values are significantly different at $P<0.01$. blooming after only $35 \mathrm{~d}$ in the light chamber, while the antisense transgenic lines remained in their vegetative phase.

After $42 \mathrm{~d}$ of culturing, floral stems from the antisense transgenic lines were $37 \%$ shorter, had diameters that were $28 \%$ smaller, and had toughness values that were $15 \%$ lower when compared with WT plants

\section{GbUGD6 enzymatic activities and qRT-PCR results for selected fiber-related genes}

qRT-PCR profiles for GbUGD6 showed that transcript levels wereabout $50 \%$ lower in the antisense transgenic lines than in the WT (Figure 3A). Our assays indicated that endogenous UGD activity was $30 \%$ to $50 \%$ lower in the antisense transgenic lines than in the WT (Figure 3B). The transgenic lines had higher cellulose contents but lower levels of UGP-GlcA than the WT (Figure 3C). For the NSO pathway genes $A t U G P 1, A t U G P 2$, and AtPGM2, expression was greatly reduced in the antisense transgenic lines. However, the MIO pathway genes AtMIOX4, AtMIPSI, and AtIMP exhibited increased expression in the antisense transgenic plants. The UGD downstream gene AtUXS was decreased, while $A t G A E$ was increased (Figure 3D). These findings suggested that GbUGD6 has a role in regulating
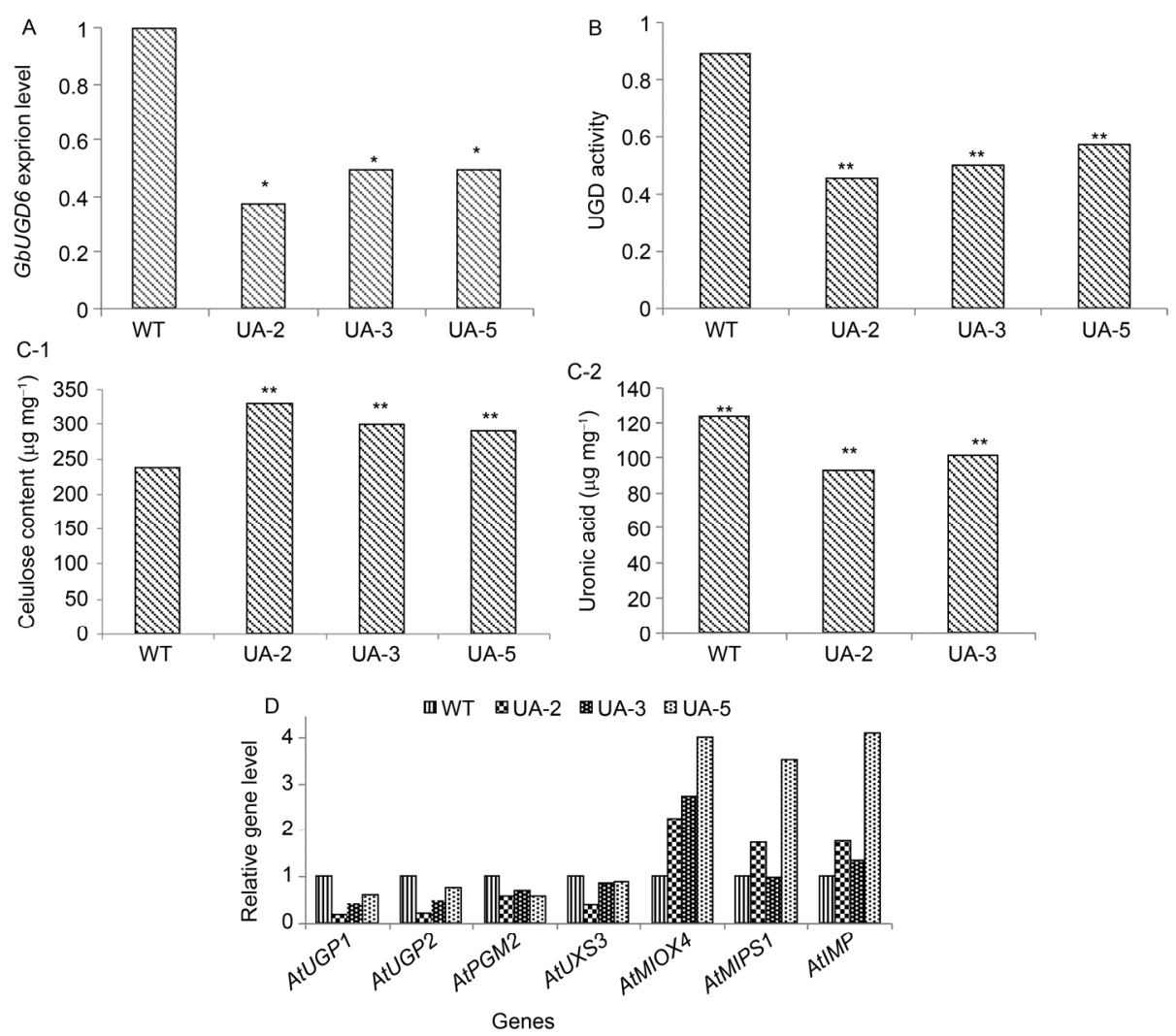

Figure 3 Characterization of antisense Arabidopsis plants with lower UGD6 expression. A, Results from real-time PCR analysis showing relative expression level for GbUGD6 in wild-type (WT) and GbUGD6-antisense (UA) plants of Arabidopsis. **, significantly different at $P<0.01$. B, Analysis of endogenous UGD activity in wild-type (WT) and GbUGD6-antisense (UA) plants of Arabidopsis. **, significantly different at $P<0.01$. C, Contents of cellulose (C-1) and UDP-GlcA (C-2) in cell walls from wild-type (WT) and GbUGD6-antisense (UA) plants of Arabidopsis. D, Results from real-time PCR analysis for transcripts of genes related to genes sugar metabolism in wild-type (WT) and GbUGD6-antisense (UA) plants of Arabidopsis. **, significantly different at $P<0.01$. 
the synthesis of polysaccharides.

\section{DISCUSSION}

UGD is the key enzyme for generating UDP-GlcA, the primary precursor of hemicelluloses and pectin polysaccharide in plant cell walls. Its functions have been investigated in many plants, including soybean, poplar, tobacco, and Arabidopsis. In the current study, we examined the UGD from Gossypium barbadense and confirmed that the enzyme participates in pathways that are involved in polysaccharide synthesis, as has been found for UGDs from other species.

Expression of GbUGD6 was detected in several stages of cotton fiber development, especially during the formation of primary and secondary cell walls. Our observations support previous reports that have implicated $U G D 1$ in cotton fiber elongation (Pang et al., 2010) and clearly demonstrate that GbUGD6 is involved in the pathways of polysaccharide synthesis in cotton. The functions of GbUGD6 were further verified using antisense transgenic Arabidopsis plants. We noted that plants from GbUGD6 antisense transgenic lines had defective growth, which is in agreement with earlier studies that have shown that an Arabidopsis mutant (ugd2,3) that lacks two of four UGD isoforms has a strong dwarfed phenotype and severe root defects (Reboul et al., 2011). The results from our expression analysis and examination of cell wall components also provide evidence that UGDs with different plant origins can have similar effects on phenotype.

GbUGD6 is critical for function of the NSO and MIO pathways, and consequently, we observed dwarfing and reduced expression of $A t U G P$ and AtPGM2 in our antisense transgenic lines. Similar to the dwarfism observed in our transgenic lines, a decrease in PGM activity can negatively influence plant growth rates and carbohydrate partitioning, and overexpression of G. hirsutum or Larix gmelinii UGPs in Arabidopsis has been shown to enhance vegetative growth and cellulose biosynthesis (Li et al., 2014; Malinova et al., 2014; Wang et al., 2011).

Furthermore, lines, expression of AtMIOX, AtMIPS, and AtIMP was up-regulated in the MIO pathway, in accord with previous studies on MIOX induction (Reboul et al., 2011). Our findings demonstrate that GbUGD6 expression is able to alter the MIO pathway and reduce the amount of UDP-GlcA that can be synthesized from UGD. When that pathway is limited, then NSO may become the key pathway for forming UDP-GlcA. This is consistent with a previous report by Reboul et al. (Reboul et al., 2011). As the common precursor for synthesizing cell wall polysaccharides, such as pectin and hemicellulose, reduced levels of UDP-GlcAwould result in a decrease in cellulose contents in transgenic seedlings.

In Arabidopsis mutants, down-regulation of GbUGD6 led to a decline in the expression of AtUXS, a gene downstream of $U G D$. Microarray analysis has shown that tran- scripts of $U G D I$ and $U X S$ are significantly reduced during the elongation stage in G. hirsutum Li2 fibers (Naoumkina et al., 2013). Moreover, UGP, UXS, and UGD are downregulated during that stage in the $f l$ mutant of the same species (Padmalatha et al., 2012).

In summary, our results indicate that the cotton gene, GbUGD6, is involved in polysaccharide and cell wall biosynthesis. Therefore, this gene may represent a valuable target for schemes to improve the cellular development of cotton fibers. Whole genome sequencing of cotton provides a chance for fiber development research (Cao, 2015). So the UGD function will be more and more clear.

\section{MATERIALS AND METHODS}

\section{Plant materials}

Plants of Gossypium hirsutum "CCRI 8" and G. barbadense "Pima90-53" were grown in an experimental field in Baoding, China. Ovules and epidermal fibers were collected at $0,5,10,15,20,25,30,35$, and 40 DPA, respectively, and were immediately immersed in liquid nitrogen.

Leaf, stem and root tissues Arabidopsis ("Columbia" ecotype, or "Col-O") seedlings were sampled from light chamber-grown (16 h light period, $23^{\circ} \mathrm{C} ; 8 \mathrm{~h}$ dark period, $22^{\circ} \mathrm{C} ; 70 \%$ relative humidity) and placed in liquid nitrogen.

Total RNAs were isolated from the ovules and fibers of both cotton cultivars with RNAplant Kits (TIANGEN, China), while RNA was isolated from Arabidopsis tissues with a TransZol Kit (TransGen Biotech, China). Purified RNA was treated with DNase I (Promega, USA), according to the manufacturer's instructions. Then, cDNA was synthesized using an M-MLV Kit (TaKaRa, China).

\section{Subcellular localization of GhUGD and GFP fusion proteins expressed in onion epidermal cells}

In order to test the subcellular localization of GbUGD6, the coding region of GbUGD6 was inserted into the pCamE: GFP expression vector to generate a pCamE-GbUGD6:: GFP fusion protein with green fluorescent protein (GFP). The correctly sized recombinant pCamE-GbUGD6::GFP plasmid and a control pCamE-GFP plasmid were transferred into living onion epidermal cells with a gene gun (PDS-1000/He Particle Delivery System; Bio-Rad, USA), according to the manufacturer's instructions. The transformed cells were incubated on Murashige and Skoog (MS) agar plates in the dark at $25^{\circ} \mathrm{C}$ for $48 \mathrm{~h}$ before being observed under a fluorescence microscope (BX51; Olympus, Japan) to detect GFP signals.

\section{Real-time PCR of GhUGD in cotton fiber cDNA}

Real-time quantitative PCR was performed with a Roche LightCycler 1.5 (Germany) in order to compare the GbUGD6 expression characters of G. hirsutum "CCRI 8" and G. barbadense "Pima90-53" fibers. Cotton EF1 $\alpha$ 
(GenBank Accession No. DQ174251.1) was amplified as the reference gene and $2 \times$ SYBR ${ }^{\circledR}$ Premix Ex Taq ${ }^{\mathrm{TM}}$ II (Perfect Real Time; TaKaRa, China) was used. No-template controls were also used for each primer pair. Each sample had three replicates. Data were examined by the $2^{-\Delta \Delta C_{\mathrm{T}}}$ value method.

The PCR mixture contained $1 \mu \mathrm{L}$ of diluted cDNA, 10 $\mu \mathrm{L}$ of $2 \times \mathrm{SYBR} \AA$ Premix Ex Taq ${ }^{\mathrm{TM}}$ II (Perfect Real Time; TaKaRa, China), and $20 \mu \mathrm{mol} \mathrm{\textrm {L } ^ { - 1 }}$ of each gene-specific primer in a final volume of $20 \mu \mathrm{L}$. Conditions for all PCR procedures included 40 cycles of $10 \mathrm{~s}$ at $95^{\circ} \mathrm{C}, 10 \mathrm{~s}$ at $60^{\circ} \mathrm{C}$, and $20 \mathrm{~s}$ at $72^{\circ} \mathrm{C}$

\section{Construction of antisense vectors and Arabidopsis transformation}

To construct our antisense pBI121p35S::AUGD6 expression vector, we amplified the entire coding region of GbUGD6 via PCR. After ligation into the vector pBI121, the integrated constructs were introduced into Agrobacterium tumefaciens strain GV3101 and transferred into WT control plants of Col-O Arabidopsis by the floral dip method (Clough and Bent, 1998).

Transgenic plants were selected on a one-half MS medium containing $100 \mathrm{mg} \mathrm{L}^{-1}$ kanamycin and were confirmed by PCR. Positive transformants were transferred to soil and allowed to set seed. The $\mathrm{T}_{3}$ generation of those transgenic plants was used for further investigations.

\section{Real-time PCR analysis and phenotypic examinations of transgenic Arabidopsis plants}

Seeds of positive $\mathrm{T}_{3}$ Arabidopsis transgenic lines, along with the WT controls, were germinated on plates containing non-selective $1 / 2 \mathrm{MS}$ medium. Roots were measured when seedlings were one week old, and phenotypic characteristics, UGD activity, and cellulose and uronic acid content were also recorded after six weeks of development. Cell wall samples were obtained from WT and transgenic Arabidopsis whole plants according to the method of Diet et al (Diet et al., 2006). The tissues were homogenized in liquid nitrogen, extracted in a mixture of $70 \%$ ethanol and chloroform/methanol $(1 / 1, v / v)$, and then washed with $100 \%$ acetone. The starch was hydrolyzed with $\alpha$-amylase and DMSO, and the samples were vacuum-dried.

Total cellulose was extracted from the cell walls as described previously (Updegraff, 1969). The levels of UGP-GlcA were quantified by a slightly modified meta-hydroxydiphenyl method (Bindschedler et al., 2005).

The UGD enzyme was extracted from 42-day-old seedlings of Arabidopsis. Activity by UGD was assayed as described by Robertson et al. (Robertson et al., 1996).

In addition, the expression of various genes in the NSO and MIO pathways were examined in antisense Arabidopsis. RT-PCR was monitored for AtUXS3 (At5g59290), AtUGP1 (At5g17310), AtUGP2 (At3g03250), AtPGM2
(At1g23190), AtMIOX4 (At4g262600), AtMIPS1 (At4g39800), AtIMP (At3g02870), and AtGAE6 (At3g23820), and Arabidopsis Ubiquitin5 was amplified as our reference gene. All primers are listed in Table S1.

Compliance and ethics The author(s) declare that they have no conflict of interest.

Acknowledgements This research was supported by the Ministry of Agriculture of China (2014ZX08009-003), the Hebei Province Technology Support Program (14962905D), and the Hebei Province Department of Education Fund (Y2012025).

Bindschedler, L.V., Wheatley, E., Gay, E., Cole, J., Cottage, A., and Bolwell, G.P. (2005). Characterisation and expression of the pathway from UDP-glucose to UDP-xylose in differentiating tobacco tissue. Plant Mol Biol 57, 285-301.

Clough, S.J., and Bent, A.F. (1998). Floral dip: a simplified method for Agrobacterium-mediated transformation of Arabidopsis thaliana. Plant J 16, 735-743.

Diaz-De-Leon, F., Klotz, K.L., and Lagrimini, L.M. (1993). Nucleotide sequence of the tobacco (Nicotiana tabacum) anionic peroxidase gene. Plant Physiol 101, 1117-1118.

Diet, A., Link, B., Seifert, G.J., Schellenberg, B., Wagner, U., Pauly, M., Reiter, W.D., and Ringli, C. (2006). The Arabidopsis root hair cell wall formation mutant $\operatorname{lr} x 1$ is suppressed by mutations in the RHM1 gene encoding a UDP-L-rhamnose synthase. Plant cell 18, 1630-1641.

Johansson, H., Sterky, F., Amini, B., Lundeberg, J., and Kleczkowski, L.A. (2002). Molecular cloning and characterization of a cDNA encoding poplar UDP-glucose dehydrogenase, a key gene of hemicellulose/pectin formation. Biochim Biophys Acta 1576, 53-58.

Karkonen, A., Murigneux, A., Martinant, J.P., Pepey, E., Tatout, C., Dudley, B.J., and Fry, S.C. (2005). UDP-glucose dehydrogenases of maize: a role in cell wall pentose biosynthesis. Biochem J 391, 409-415.

Klinghammer, M., and Tenhaken, R. (2007). Genome-wide analysis of the UDP-glucose dehydrogenase gene family in Arabidopsis, a key enzyme for matrix polysaccharides in cell walls. J Exp Botany 58, 3609-3621.

Kuhn, J.H., Becker, S., Ebihara, H., Geisbert, T.W., Johnson, K.M., Kawaoka, Y., Lipkin, W.I., Negredo, A.I., Netesov, S.V., Nichol, S.T., Palacios, G., Peters, C.J., Tenorio, A., Volchkov, V.E., and Jahrling, P.B. (2010). Proposal for a revised taxonomy of the family Filoviridae: classification, names of taxa and viruses, and virus abbreviations. Arch Virol 155, 2083-2103.

Li, N., Wang, L., Zhang, W., Takechi, K., Takano, H., and Lin, X. (2014). Overexpression of UDP-glucose pyrophosphorylase from Larix gmelinii enhances vegetative growth in transgenic Arabidopsis thaliana. Plant Cell Rep 33, 779-791.

Malinova, I., Kunz, H.H., Alseekh, S., Herbst, K., Fernie, A.R., Gierth, M., and Fettke, J. (2014). Reduction of the cytosolic phosphoglucomutase in Arabidopsis reveals impact on plant growth, seed and root development, and carbohydrate partitioning. PLoS One 9, e112468.

Naoumkina, M., Hinchliffe, D.J., Turley, R.B., Bland, J.M., and Fang, D.D. (2013). Integrated metabolomics and genomics analysis provides new insights into the fiber elongation process in Ligon lintless- 2 mutant cotton (Gossypium hirsutum L.). BMC Genomics 14, 155.

Padmalatha, K.V., Patil, D.P., Kumar, K., Dhandapani, G., Kanakachari, M., Phanindra, M.L., Kumar, S., Mohan, T.C., Jain, N., Prakash, A.H., Vamadevaiah, H., Katageri, I.S., Leelavathi, S., Reddy, M.K., Kumar, P.A., and Reddy, V.S. (2012). Functional genomics of fuzzless-lintless mutant of Gossypium hirsutum L. cv. MCU5 reveal key genes and pathways involved in cotton fibre initiation and elongation. BMC Genomics 13, 624.

Pan, Y., Ma, J., Zhang, G., Han, G., Wang, X., and Ma, Z. (2007). 
cDNA-AFLP profiling for the fiber development stage of secondary cell wall synthesis and transcriptome mapping in cotton. Chin Sci Bull 52, 2358-2364.

Pang, C., Wang, H., Pang, Y., Xu, C., Jiao, Y., Qin, Y., Western, T.L., Yu, S., and Zhu, Y. (2010). Comparative proteomics indicates that biosynthesis of pectic precursors is important for cotton fiber and Arabidopsis root hair elongation. Mol Cell Proteomics 9, 2019-2033.

Reboul, R., Geserick, C., Pabst, M., Frey, B., Wittmann, D., Lutz-Meindl, U., Leonard, R., and Tenhaken, R. (2011). Down-regulation of UDP-glucuronic acid biosynthesis leads to swollen plant cell walls and severe developmental defects associated with changes in pectic polysaccharides. J Biol Chem 286, 39982-39992.

Robertson, D., Smith, C., and Bolwell, G.P. (1996). Inducible UDP-glucose dehydrogenase from French bean (Phaseolus vulgaris L.) locates to vascular tissue and has alcohol dehydrogenase activity. Biochem J 313, 311-317.

Seifert, G.J. (2004). Nucleotide sugar interconversions and cell wall biosynthesis: how to bring the inside to the outside. Curr Opin Plant Biol 7, 277-284.

Tenhaken, R., and Thulke, O. (1996). Cloning of an enzyme that synthesizes a key nucleotide-sugar precursor of hemicellulose biosynthesis from soybean: UDP-glucose dehydrogenase. Plant Physiol $112,1127-1134$.

Updegraff, D.M. (1969). Semimicro determination of cellulose in biological materials. Anal Biochem 32, 420-424.

Wang, Q., Zhang, X., Li, F., Hou, Y., Liu, X., and Zhang, X. (2011). Identification of a UDP-glucose pyrophosphorylase from cotton (Gossypium hirsutum L.) involved in cellulose biosynthesis in Arabidopsis thaliana. Plant Cell Rep 30, 1303-1312.

Zablackis, E., Huang, J., Muller, B., Darvill, A.G., and Albersheim, P. (1995). Characterization of the cell-wall polysaccharides of Arabidopsis thaliana leaves. Plant Physiol 107, 1129-1138.

Cao, X. (2015). Whole genome sequencing of cotton-a new chapter in cotton genomics. Sci China Life Sci 58, 515-516.

Open Access This article is distributed under the terms of the Creative Commons Attribution License which permits any use, distribution, and reproduction in any medium, provided the original author(s) and source are credited.

\section{SUPPORTING INFORMATION}

Figure S1 Nucleotide and deduced amino acid sequences of GbUGD6, with domain underlined and alignment of UGD domain from GbUGD6 with that from other closely related proteins

Figure S2 Patterns of relative expression by UGD6 over time in stem fibers from Gossypium hirsutum cv. CCRI 8 and G. barbadense cv. Pima90-53. DPA, days post-anthesis

Table S1 Primers used in RT-PCR analysis of transgenic Arabidopsis.

The supporting information is available online at life.scichina.com and link.springer.com. The supporting materials are published as submitted, without typesetting or editing. The responsibility for scientific accuracy and content remains entirely with the authors. 